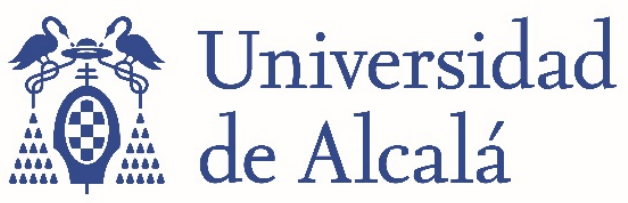

BIBLIOTECA

Document downloaded from the institutional repository of the University of Alcala: http://dspace.uah.es/dspace/

This is the peer reviewed version of the following article:

Righetti, P.G., Esteve, C., D'Amato, A., Fasoli, E., Luisa Marina, M. \& Concepción García, M. 2015, "A sarabande of tropical fruit proteomics: Avocado, banana, and mango", Proteomics, vol. 15, no. 10, pp. 1639-1645,

which has been published in final form at:

http://dx.doi.org/10.1002/pmic.201400325

This article may be used for non-commercial purposes in accordance with Wiley Terms and Conditions for Self-Archiving

(C) 2015 Wiley

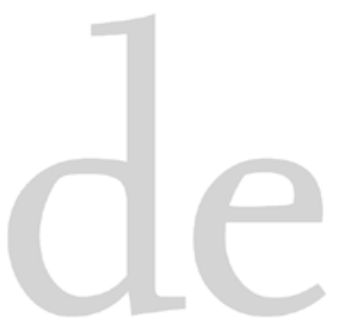

(Article begins on next page)

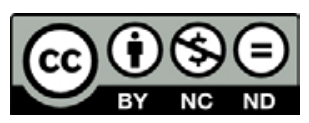

This work is licensed under a

Creative Commons Attribution-NonCommercial-NoDerivatives

4.0 International License. 


\section{A sarabande of tropical fruit proteomics: Avocado, banana and mango}

Pier Giorgio Righetti ${ }^{1}$, Clara Esteve ${ }^{2}$, Alfonsina D’Amato ${ }^{1}$, Elisa Fasoli ${ }^{1}$, María Luisa

Marina $^{2}$, María Concepción García

${ }^{1}$ Department of Chemistry, Materials and Chemical Engineering "Giulio Natta", Politecnico di Milano, 20131 Milan, Italy.

${ }^{2}$ Department of Analytical Chemistry, Faculty of Chemistry, University of Alcalá, Ctra. Madrid-Barcelona, Km. 33.600, 28871 Alcalá de Henares, Madrid, Spain,

Correspondence: Professor Pier Giorgio Righetti, Department of Chemistry, Materials and Chemical Engineering "Giulio Natta", Politecnico di Milano, 20131 Milan, Italy

E-mail: piergiorgio.righetti@polimi.it, Fax: +39-02-23993080

Abbreviations: CPLL, combinatorial peptide ligand libraries; HAP, high abundance proteins; LAP, low abundance proteome

Keywords: Low abundance proteome / Tropical fruits / Allergens / Combinatorial peptide ligand libraries / Mass spectrometry 


\section{Summary}

The present review highlights the progress made in plant proteomics via the introduction of combinatorial peptide ligand libraries (CPLL) for detecting lowabundance species. Thanks to a novel approach to the CPLL methodology, namely that of performing the capture both under native and denaturing conditions, identifying plant species in the order of thousands, rather than hundreds, is now possible. We report here data on a trio of tropical fruits, namely banana, avocado and mango. The first two are classified as "recalcitrant" tissues since minute amounts of proteins (in the order of $1 \%$ ) are embedded on a very large matrix of plant-specific material (e.g., polysaccharides and other plant polymers). Yet, even under these adverse conditions we could report, in a single sweep, from 1000 to 3000 unique gene products. In the case of mango the investigation has been extended to the peel too, since this skin is popularly used to flavour dishes in Far East cuisine. Even in this tough peel 330 proteins could be identified, whereas in soft peels, such lemons, one thousand unique species could be detected.

\section{Introduction}

The present review aims at offering a panorama of what modern pre-fractionation technologies can achieve in detecting the low- to very-low abundance proteins (LAP) in plant proteomics, an analyte fraction that is quite invisible even to the most sophisticated modern MS, whose sensitivity spans in general five orders of magnitude in relative concentrations of proteins present in a sample. Yet, in human biological fluids, such as plasma, such a dynamic range can cover up to 12 orders of magnitude [1] and, in living cells, it can span at least seven orders of magnitude. Thus, it is quite obvious that MS alone cannot efficiently cover the ground and thus additional techniques would be needed to achieve the goal of (hopefully) a global coverage of any proteome. One such technique, which will be reviewed here with examples of what can be achieved in analysis of plant proteomics, is definitely the combinatorial peptide ligand library (CPLL) technology. This technology has been developed over the years and it has now been taken at a level of maximum performance, as summarized in a recent book [2]. 
As just stated, CPLLs appear to be a unique tool for exploring the "dark side" of any proteome, due to their unique property of providing millions of affinity ligands able to find a partner in any protein species present in biological materials. It must be emphasized that in just about any biological specimen a small set of proteins (often as few as 20-30) are present in large excess and they could constitute, like in human sera, as much as $99 \%$ of the total protein mass. This would leave little room for sampling (and thus detecting) all other species present therein. A solution proposed already in 2002 by the Anderson's lab was immuno-subtraction, i.e., preparation of affinity resins containing antibodies against the six most abundant proteins in sera (first extended to 12 and now to 20) [3]. It was believed to permit access to low-abundance species [4], but in reality it did not quite live up to expectations $[5,6]$ for several reasons. Among them, the major issue was that far too little sample volume could be processed in a single sweep (barely $100 \mu \mathrm{L}$ serum/plasma). Application of this methodology to plant proteomes, such as the immuno-depletion of RuBisCO, also did not lead to any major improvement [7]. The CPLL technique that we have developed is immune to such drawbacks. To start with, these beads can be loaded with any volume (and quantity) of sample, since they work on an overloading principle. Additionally, they are universal, since they can be applied to any sample of any origin, whereas immune-subtraction relies on antibodies made against specific samples, which involves preparing a new one when the organism is changed. Thirdly, and most importantly, these beads act simultaneously by drastically cutting the concentration of high-abundance species while enriching and concentrating the LAP population to the maximum possible extent (i.e. as long as there are species in solution, depending on how large the sample applied is). The consequence of such a situation is the detection of many novel proteins (low-abundance) as a result of (i) the annihilation of the signal suppression due to concentrated species (e.g., albumin in serum) and (ii) the detection of very low concentration proteins that were below the detectability level prior to sample treatment [8-10]. Although we have discussed and reviewed the properties and mechanism of action of CPLLs several times, we will briefly report them again here. CPLLs comprise several million hexapeptides (probably made by 
using 16 different amino acids) which are potentially able to recognize a complementary amino acid sequence in a bait protein, thus harvesting it from the sample matrix. Therefore CPLLs can be envisioned as a matrix consisting of millions of bio-affinity ligands, contrary to classical affinity chromatography where, in general, a single ligand specific for a given protein is bound to a resin [2]. Their ability to capture a given protein, especially if present in very low abundance (LAP), as compared to much more concentrated proteins (HAP), depends on the relative affinities for a given bait. Thus, a LAP, having very high affinity for a given hexapeptide, can displace from it an HAP having low affinity for the same ligand. This mechanism of action can thus counterbalance the law of mass action and permit capture and much increased visibility of LAPs in the presence of HAPs.

We have applied the CPLL technique to plant proteomics (but in particular to analysis of foodstuffs and beverages of plant and animal origin) for three main reasons:

(i) To detect trace proteins/peptides exhibiting negative effects on health (e.g., allergens);

(ii) To detect trace proteins/peptides displaying positive effects on health (e.g., anti-microbial, anti-hypertensive and anti-oxidant activities);

(iii) To expose fraud in commercial food products and provide a proof of genuineness for "correct" commercial foods, as found in supermarkets.

The present review is limited to application of the CPLL methodology to three typical tropical fruits of very large consumption, namely the banana, avocado and mango, whose proteome was largely unknown before our investigations. Additionally, two of them (avocado and banana) represent "recalcitrant tissues" in that minute amounts of proteins (of the order of $1 \%$ ) are embedded on a very large matrix of plant-specific material (e.g., polysaccharides and other plant polymers). Thus, the description and discovery of their proteomes represents a real challenge. Therefore, we will not cover here the vast literature on plant and food proteomics, but we will just mention two recent reviews (and references therein) covering this field quite extensively $[11,12]$. Special issues of different journals appear from time to time covering plant proteomics, including analysis of food and beverages. A recent This article is protected by copyright. All rights reserved. 
one has been released by Journal of Proteomics [13]. Within this issue see, for instance, Boggess et al. [12], Nakamura et al. [14], Uvackova et al. [15] and Agrawal et al. [16].

\section{Mammalian versus plant proteomics}

When surveying the deeds of scientists working with mammalian proteomics, it is clear that today they can explore to an incredible extent the proteome of any living cell line. For instance, when analyzing 11 human cell lines, Geiger et al. [17] could identify a total of 11,731 proteins and on average $10,361 \pm 120$ proteins in each cell line, an outstanding catch, indeed. Interestingly, a very large number of them represent a common set shared by all cell lines, amounting to 8522 unique species. Each individual cell then displays from 200 to 500 proteins specific of each line. The latter probably represent proteins that characterize each individual line and ensure its specific biological activity. It is likely that they could also be low-abundance species. How can there be such a discrepancy with plant proteomics, when in this last domain we are lucky if we can find a few hundred species in a single run? It should be noted that, in a way, these mammalian cell lines grown in vitro cultures are rather "easy" samples, in that they are not embedded in, e.g., fibrous tissues, muscles and other body compartments that would represent a complex matrix from which such cells would have to be extracted. On the contrary, in plant proteomics, most of the time, proteins to be identified are dispersed into a very complex matrix and often are present in low amounts as compared to the plant biopolymers (e.g., polysaccharides, polyphenols) and metabolites constituting the specimen mass. This is the reason why it has been difficult to detect more than a few hundred species in any plant tissue. Thus, the CPLL methodology described here, allowing access to an order of magnitude more (up to 3000 unique gene products), represents an important advance in the field. Clearly, however, more efforts should be dedicated to improving extraction technologies so as to match what is obtained in mammalian proteomics today. 
There is a reason, though, for this major advance in mapping of fruit proteomics.

Up to these investigations, in fact, we applied the CPLL technology under "orthodox" conditions, namely by capturing proteomes under native conditions, since it was believed (correctly) that CPLLs would not be compatible with denaturing milieus, such as the classical cocktail of $2 \mathrm{M}$ thiourea, $7 \mathrm{M}$ urea and $2 \%$ CHAPS adopted for solubilizing proteins in view of two-dimensional mapping. Such conditions, indeed, are used to elute the proteins captured by CPLLs, so they could hardly be applied for their capture! Yet, we found an escape route: after solubilizing proteins (and capturing them) under native conditions, the remaining sample could be treated with $2 \%$ SDS, but not under boiling conditions, only at room temperature. Under these conditions, plenty of additional species could be solubilized. Yet the presence of $2 \%$ SDS would be incompatible with further sample treatment via CPLLs. However, by diluting the sample to barely $0.1 \%$ SDS and adding a larger excess of surfactants compatible with CPLLs (up to $1 \%$ of Triton X 100 or CHAPS) this denatured sample is now amenable to CPLL capture. This is illustrated in Fig. 1. Thus, by subjecting the sample to this double capture, under native and denaturing conditions, the number of proteins that can be identified increases substantially to well above 1000 species, a major increment over the past. Of course there are more ways for both capturing and eluting proteins from CPLL beads. They cannot possibly be reviewed here; however, in the book by Boschetti and Righetti [2], in Chapter 8, no less than 56 pages are devoted to "Detailed Methodologies and Protocols" (among them, notably, the fact that it is possible to digest the harvested proteins directly on the CPLL beads, collect the peptides and go directly to MS analysis). Readers are thus referred to this book for detailed treatments on methodologies related to CPLLs.

\section{$3 \quad$ Banana proteomics}

The first tropical fruit investigated by us, among the "recalcitrant tissues", was the banana. Musa spp., comprising banana and plantain, is grown extensively in many developing countries and is considered to be one of the most important sources of energy in the diet of people living in tropical humid regions. Due to its antioxidant and This article is protected by copyright. All rights reserved. 
cell anti-proliferative activities, the consumption of banana has been associated with reduced risk of chronic diseases such as cardiovascular diseases and cancer [18]. To date, no in-depth work has focused on identifying the banana fruit proteome; since fresh banana pulp contains approximately $20 \%$ carbohydrates and only $1 \%$ proteins. This fruit has been traditionally considered as a difficult matrix for protein extraction, being a target in studies of optimization of protein extraction methodologies [19]. Fruits, as every biological source, contain HAP, which are often of limited interest for proteome analysis, whereas other proteins may be less abundant in orders of magnitude, although still of high importance. Here too, by using CPLLs, advanced mass spectrometry techniques and Musa mRNAs database in combination with Uniprot_viridiplantae database, we could identify, for the first time, 1131 proteins [20]. Among the large amount of species found, several already known allergens such as musa a 1, pectinesterase, superoxide dismutase and potentially new allergens have been detected. Additionally, several enzymes involved in degradation of starch granules and strictly correlated to ripening stage were identified. These results constitute the largest description so far of the banana proteome. Figure 2 summarizes the data discussed here. The upper left gel strips represent the SDS-PAGE profiling of a sample prior and after CPLL capture. The Venn diagrams exhibit the protein IDs as obtained in the control and in the CPLL treated sample and using CPLLs under native and denatured conditions. The graph on the right displays the major GO categories in which the various species have been classified. The use of CPLLs more than doubled (from 452 to 1131) the number of identified proteins.

\section{$4 \quad$ Avocado proteomics}

Avocado, the fruit of the tropical tree Persea americana, native to Mexico, is nowadays grown and consumed in many parts of the world. The oil obtained from pressing the avocado fruit, already used in Mexican folk medicine in the XVI century [21], is nowadays employed for manufacturing foodstuffs, cosmetics and health care products [22]. Besides this, avocado oil has been proposed as a domestic source of cooking oil to help improve the nutritional status of populations in some developing countries. The consumption of both the avocado fruit and oil has been associated 
with health benefits such as the decrease of total serum cholesterol, LDL-cholesterol and triglycerides [23], the control of blood pressure and the inhibition of certain types of cancer [24]. The avocado fruit composition has been deeply studied, leading to good characterization of small-size compounds such as fatty acids and sterols. However, up to the present, no work had been focused on identifying the avocado pulp proteome; only some reports have appeared on the avocado seed [25] and root [26] proteins, with a total identification of proteins of the order of a few dozens.

On the contrary, by using the CPLL approach, the total number of unique gene products (meaning single, non-redundant proteins, excluding isoforms, posttranslations modifications, truncated forms etc.) identified amounted to 1012 proteins, of which 174 were in common with the control, untreated sample, 190 were present only in the control and 648 represented the new species detected via CPLLs of all combined eluates, likely representing low-abundance proteins. Among the 1012 proteins, it was possible to identify the already known avocado allergen Pers a 1 and different proteins susceptible to be allergens like a profilin, a polygalacturonase, a thaumatin-like protein, a glucanase, and an isoflavone reductase-like protein. Figure 3 summarizes the data discussed here. The upper left gel strips represent the SDS-PAGE profiling of the samples prior to and after CPLL capture. The Venn diagrams exhibit the protein IDs as obtained in the control and in the CPLLs treated sample and using CPLLs under native and denatured conditions. The graph on the right displays the major GO categories in which the various species have been enriched. In this case, application of the CPLLs technique almost tripled (from 364 to 1012) the number of identified proteins, many of them observed under denaturing conditions. Moreover, it can be appreciated that, in all GO categories, the number of protein species identified after CPLL capture are much higher than in the controls. Additionally, in the CPLL eluates, three novel categories, not represented in the control, could be detected [27].

\section{$5 \quad$ Mango proteomics}

The mango is a fleshy stone fruit belonging to the genus Mangifera, consisting of numerous tropical fruiting trees in the flowering plant family Anacardiaceae 


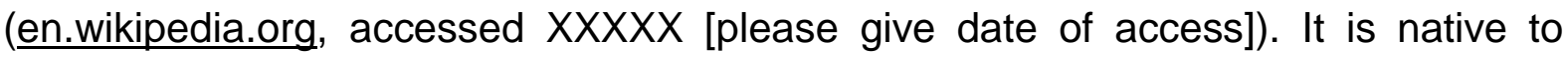
South Asia and has spread worldwide to become one of the most widely cultivated fruits in the tropics. Among the different species Mangifera indica - the 'common mango' or 'Indian mango' - is the only mango tree commonly cultivated in many tropical and subtropical regions. It is the national fruit of India, Pakistan and the Philippines, and the national tree of Bangladesh. The ripe fruit varies in size and colour. Cultivars are typically yellow, orange, red or green, and carry a single flat, oblong pit that can be fibrous or hairy on the surface, and which does not separate easily from the pulp. Mango is used to make juices, smoothies, ice cream, fruit bars, raspados, aguasfrescas, pies and sweet chili sauce, or mixed with chamoy, a sweet and spicy chili paste (en.wikipedia.org, accessed XXXXX [please give date of access]). It is popular on a stick dipped in hot chili powder and salt or as a main ingredient in fresh fruit combinations. In Central America, mango is either eaten green mixed with salt, vinegar, black pepper and hot sauce, or ripe in various forms. Some people also add soy sauce or chili sauce. Pieces of mango can be mashed and used as a topping on ice cream or blended with milk and ice as milkshakes. Sweet glutinous rice is flavoured with coconut, then served with sliced mango as a dessert.

In mango fruit pulp, the antioxidant vitamins $A$ and $C$, vitamin B6 (pyridoxine), folate, other B vitamins and essential nutrients, such as potassium, copper and amino acids, are present. Mango peel and pulp contain other compounds, such as pigment carotenoids and polyphenols, and omega-3 and -6 polyunsaturated fatty acids [28]. Additionally, mango peel pigments seem to have important biological effects [29], and include carotenoids such as the provitamin A compound, betacarotene, lutein and alpha-carotene [30], polyphenols [31] such as quercetin, kaempferol, gallic acid, caffeic acid, catechins, tannins, and the unique mango xanthonoid, mangiferin [32], all of which may counteract free radicals in various disease processes. Notwithstanding all the data reported above on mango metabolites, not much is known about this fruit proteome. Only recently, a 2D mango pulp analysis has been reported, aiming at identifying modulation of protein expression associated with ripening [33]. A total of 373 spots could be visualized in 
the 2D map, leading to the identification of 51 unique gene products. In another report, via database searches of mango-derived ESTs and proteins along with proteins from six other closely related plant species, Renuse et al. [34] could identify 1001 peptides that matched 538 proteins. However, this set of proteins applied only to the mango leaves, not to the pulp or peel.

In the case of this fruit, our research has taken a sudden twist: we also explored the proteome of the peel, since this part of the fruit is largely used in Far East cuisine to flavor plenty of dishes. Additionally, we captured its peptidome, after protein removal, via $\mathrm{C}_{18}$ resins [35]. This idea of also exploring the peel proteome of fruits has been extended to other plant samples as well. For instance, we have reported the proteome of lemon peel (the flavedo, not the albedo), since this part of the fruit is used in the Mediterranean area not only for flavouring dishes, but also for producing the very popular liqueur called, aptly, "Limoncello", indeed an infusion of the lemon flavedo [36]. Recently, we have applied this strategy also to the peels of oranges and clementines (work in progress), since these skins are also used in cuisine. By performing the capture under both native and denaturing conditions, a total of 334 unique protein species were identified in the peel vs 2855 in the pulp, by acting at two different pH values (2.2 and 7.2). These data are presented in Fig. 4, where the gel strips on the right exhibit SDS-PAGE profiles prior to and after CPLL capture and the Venn diagrams show the total species identified in both peel and pulp. Figure 5 displays the major GO categories in which the various species have been classified in both compartments. It is of interest to note that, although in the peel only slightly more than $10 \%$ of those proteins detected in the pulp are present, at least $8 \mathrm{GO}$ categories are unique to this organ and apparently absent in the pulp, suggesting that they have a special biological role confined to the skin. Conversely, in the pulp, another 8 GO categories appear which do not seem to have a counterpart in the peel. In regard to mango's potential allergies, the responsible allergens have not yet been identified, due also to the fact that the mango proteome is not completely known because its genome has not yet been sequenced. For this reason in allergen databases we could not find specific proteins regarding the mango but in our identified species we could verify the presence of well-known allergens, referring to the same plant order (Sapindales) and belonging to the same taxonomic group This article is protected by copyright. All rights reserved. 
(Plantae Magnoliopsida). By consulting the IUIS allergen nomenclature databases (http://www.allergen.org/), we obtained a list of allergens, of which some are present in our list. In particular in CPLLs eluates we recognized non-specific lipid transfer protein, superoxide dismutase, germin-like protein and profilin.

\section{Discussion}

We hope that the data briefly summarized here will show the unique potential of CPLLs in detecting those low-abundance species which sorely miss the roll call in plant proteomics. It can also be appreciated that, by using CPLLs coupled with modern MS instrumentation, an important step forward has been made in plant proteomics, permitting, in a single sweep, identifications ranging now in the thousands, vs barely in the hundreds up to recent times. Yet, we cannot ignore that mammalian proteomics is far ahead as compared to plant proteomics, since in this domain it is not uncommon to see papers reporting IDs above tens of thousands in a single run (another important reason, of course, is the fact that mammalian genomes, and especially the human genomes, have been much more extensively sequenced and annotated than those of plants). Thus, whereas mammalian proteomics can now claim to perform at the level of Beethoven Symphonies, we have to limit our music, at present, to an honest sarabande. Yet even a sarabande has found full dignity and space in the domain of music, as exemplified by the famous sarabande by Händel in the baroque period, as well as by other composers in the late $19^{\text {th }}$ and early $20^{\text {th }}$ centuries, such as Grieg, Debussy, Satie and many others. It may be that, in order to reach the level of Beethoven, plant proteomics will have to wait a little longer!

Conflicts of Interest

The authors have declared no conflict of interest 


\section{7} References

[1] Anderson, N.L., Anderson, N.G. The human plasma proteome: History, character, and diagnostic prospects. Mol. Cell. Proteomics 2002, 1, 845-867.

[2] Boschetti, E., Righetti, P.G. Low-Abundance Protein Discovery: State of the Art and Protocols; Elsevier: Amsterdam, 2013, pp. 1-345.

[3] Pieper, R., Gatlin, C.L., Makusky, A.J., Russo, P.S., Schatz, C.R., Miller, S.S., Su, Q., McGrath, A.M., Estock, M.A., Parmar, P.P., et al. The human serum proteome: display of nearly 3700 chromatographically separated protein spots on two-dimensional electrophoresis gels and identification of 325 distinct proteins. Proteomics 2003, 3, 1345-1364.

[4] Fang, X., Zhang, W.W. Affinity separation and enrichment methods in proteomic analysis. J. Proteomics 2008, 71, 208-303.

[5] Tu, C.; Rudnick, P.A.; Martinez, M.Y.; Cheek, K.L.; Stein, S.E.; Slebos, R.J.; Liebler, D.C. Depletion of abundant plasma proteins and limitations of plasma proteomics. J. Proteome Res. 2010, 9, 4982-4991.

[6] Shen, Y.;Kim, J.; Strittmatter, E.F.; Jacobs, J.M.; Camp, D.G., 2nd; Fang, R.; Tolié, N.; Moore, R.J.; Smith, R.D. Characterization of the human blood plasma proteome. Proteomics 2005, 5, 4034-4045.

[7] Widjaja, I., Naumann, K., Roth, U., Wolf, N., Mackey, D., Dangl, J.L. Scheel, D., Lee, J. Combining sub-proteome enrichment and RuBisCO depletion enables identification of low abundance proteins differentially regulated during plant defense. Proteomics 2009, 9, 138-147. 
[8] Boschetti, E., Lomas, L., Citterio, A., Righetti, P.G. Romancing the "hidden proteome", Anno Domini two zero zero six. J. Chromatogr. A 2007, 1153, $277-290$

[9] Righetti, P.G., Boschetti, E., Lomas, L., Citterio, A. Protein Equalizer technology: The quest for a "democratic proteome". Proteomics 2006, 6, 3980-3992.

[10] Righetti, P.G., Boschetti, E., Kravchuk, A.V., Fasoli, E. The proteome buccaneers: How to unearth your treasure chest via combinatorial peptide ligand libraries. Expert Rev. Proteomics 2010, 7, 373-385.

[11] Agrawal, G.K., Sarkar, A., Righetti, P.G., Pedreschi, R., Carpentier, S., Wang, T., Barkla, B.J., Kohli, A., Ndimba, B.K., Bykova, N.V. et al. A decade of plant proteomics and mass spectrometry: Translation of technical advancements to food security and safety issues. Mass Spectrom. Rev. 2013, 32, 335-365.

[12] Boggess, M.V., Lippolis, J.D., Hurkman, W.J., Fagerquist, C.K., Briggs, S.P., Gomes, A.V., Righetti, P.G., Bala, K. The need for agriculture phenotyping: "Moving from genotype to phenotype". J. Proteomics 2013, 93, 20-39.

[13] Jorrín-Novo, J., Valledor Gonzalez, L. (Guest Editors) Special Issue: Translational Plant Proteomics. J. Proteomics 2013, 93, pp. 1-368.

[14] Nakamura, R., Teshima, R. Proteomics-based allergen analysis in plants. J. Proteomics2013, 93, 40-49.

[15] Lubica Uvackova, L., Skultety, L., Bekesova, S., McClain, S., Hajduch, M. The MSE-proteomic analysis of gliadins and glutenins in wheat grain identifies and 
quantifies proteins associated with celiac disease and baker's asthma. J. Proteomics 2013, 93, 65-73.

[16] Agrawal, G.K., Timperio, A.M., Zolla, L., Bansal, V., Shukla, R., Rakwal, R. Biomarker discovery and applications for foods and beverages: Proteomics to nano-proteomics. J. Proteomics 2013, 93, 74-92.

[17] Geiger, T., Wehner, A., Schaab, C., Cox, J., Mann, M. Comparative proteomic analysis of eleven common cell lines reveals ubiquitous but varying expression of most proteins. Mol. Cell. Proteomics 2012, 11, M111.014050.

[18] Sun, J., Chu, Y.F., Wu, X., Liu, R.H. Antioxidant and anti-proliferative activities of common fruits. J. Agric. Food Chem. 2002, 50, 7449-7454.

[19] Saravanan, R.S., Rose, J.K.C. A critical evaluation of sample extraction techniques for enhanced proteomic analysis of recalcitrant plant tissues. Proteomics 2004, 4, 2522-2532.

[20] Esteve, C., D’Amato, A., Marina, M.L., Concepción García, M., Righetti, P.G. Indepth proteomic analysis of banana (Musa spp.) fruit with combinatorial peptide ligand libraries. Electrophoresis 2013, 34, 207-214.

[21] Argueta-Villamar, A., Cano, L., Rodarte, M., Atlas de las Plantas de la Medicina Tradicional Mexicana. Ed. Instituto Nacional Indigenista, México, 1994, pp. 55.

[22] Swisher, H. O., Avocado oil from food use to skin care. J. Am. Oil Chem. Soc. $1993,65,1704-1706$. 
[23] Lopez-Ledesma, R., Frati-Munari, A. C., Hernandez-Dominguez, B. C., Cervantes-Montalvo, S., Hernandez-Luna, M. H., Juarez, C., Morant-Lira, S., Monounsaturated fatty acid (avocado) rich diet for mild hypercholesterolemia. Arch. Med. Res. 1996, 27, 519-523.

[24] Lu, Q. Y., Arteaga, J. R., Zhang, Q., Huerta, S., Go, V. L. W., Heber D., Inhibition of prostate cancer cell growth by an avocado extract: role of lipidsoluble bioactive substances. J. Nutr. Biochem. 2005, 16, 23-30.

[25] Sánchez-Romero, C., Perán-Quesada, R., Barceló-Muñoz, A., Pliego-Alfaro, F., Variations in storage protein and carbohydrate levels during development of avocado zygotic embryos. Plant Physiol. Biochem. 2002, 40, 1043-1049.

[26] Acosta-Muñiz, C. H., Escobar-Tovar, L., Valdes-Rodriguez, S., FernandezPavia, S., Arias-Saucedo, L. J., de la Cruz Espindola Barquera, M. C., Gomez Lim, M. A., Identification of avocado (Persea americana) root proteins induced by infection with the oomycete Phytophthoracinnamomi using a proteomic approach. Physiol. Plant.2012, 144, 59-72.

[27] Esteve, C., D'Amato, A., Marina, M.L., García, M.C., Righetti, P.G. Identification of avocado (Persea americana) pulp proteins by nanoLC-MS/MS via combinatorial peptide ligand libraries. Electrophoresis 2012, 33, 2799-2805.

[28] Ajila, C.M., Prasada Rao, U.J. Protection against hydrogen peroxide induced oxidative damage in rat erythrocytes by Mangifera indica L. peel extract. Food Chem. Toxicol. 2008, 46, 303-309.

[29] Berardini, N., Fezer, R., Conrad, J., Beifuss, U., Carle, R., Schieber, A. Screening of mango (Mangifera indica L.) cultivars for their contents of flavonol-O - and xanthone C-glycosides, anthocyanins, and pectin. J. Agric. Food Chem. 2005, 53, 1563-1570. 
[30] Gouado, I., Schweigert, F.J., Ejoh, R.A., Tchouanguep, M.F., Camp, J.V. Systemic levels of carotenoids from mangoes and papaya consumed in three forms (juice, fresh and dry slice). Eur. J. Clin. Nutr. 2007, 61, 1180-1188.

[31] Singh, U.P., Singh, D.P., Singh, M., et al. Characterization of phenolic compounds in some Indian mango cultivars. Int. J. Food Sci. Nutr. 2004, 55, 163-169.

[32] Andreu, G.L., Delgado, R., Velho, J.A., Curti, C., Vercesi, A.E. Mangiferin, a natural occurring glucosyl xanthone, increases susceptibility of rat liver mitochondria to calcium-induced permeability transition. Arch. Biochem. Biophys. 2005, 439, 184-193.

[33] De Magalhães Andrade, J., Torres Toledo, T., Beserra Nogueira, S., Cordenunsi, B.R., Lajolo, F.M., Oliveira do Nascimento, J.R. 2D-DIGE analysis of mango (Mangifera indica L.) fruit reveals major proteomic changes associated with ripening. J. Proteomics 2012, 75, 3331-3341.

[34] Renuse, S., Harsha, H.C., Kumar, P., Acharya, P.K., Sharma, J., Goel, R., Kumar, G.S., Raju, R.,Prasad, T.S., Slotta, T., Pandey, A. Proteomic analysis of an unsequenced plant-Mangifera indica. J. Proteomics2012, 75, 57935796.

[35] Fasoli, E., Righetti, P.G. The peel and pulp of mango fruit: a proteomic samba. Biochim. Biophys. Acta 2013, 1834, 2539-2545.

[36] Fasoli, E., Colzani, M., Aldini, G., Citterio, A., Righetti, P.G. Lemon peel and Limoncello liqueur: A proteomic duet. Biochim. Biophys. Acta 2013, 1834, 1484-1491. 
Figure 1 General scheme for extraction of proteins via CPLLs under native and denaturing conditions.

Figure 1-Righetti et al.

\section{Mass Spectrometry analysis of tropical fruits}

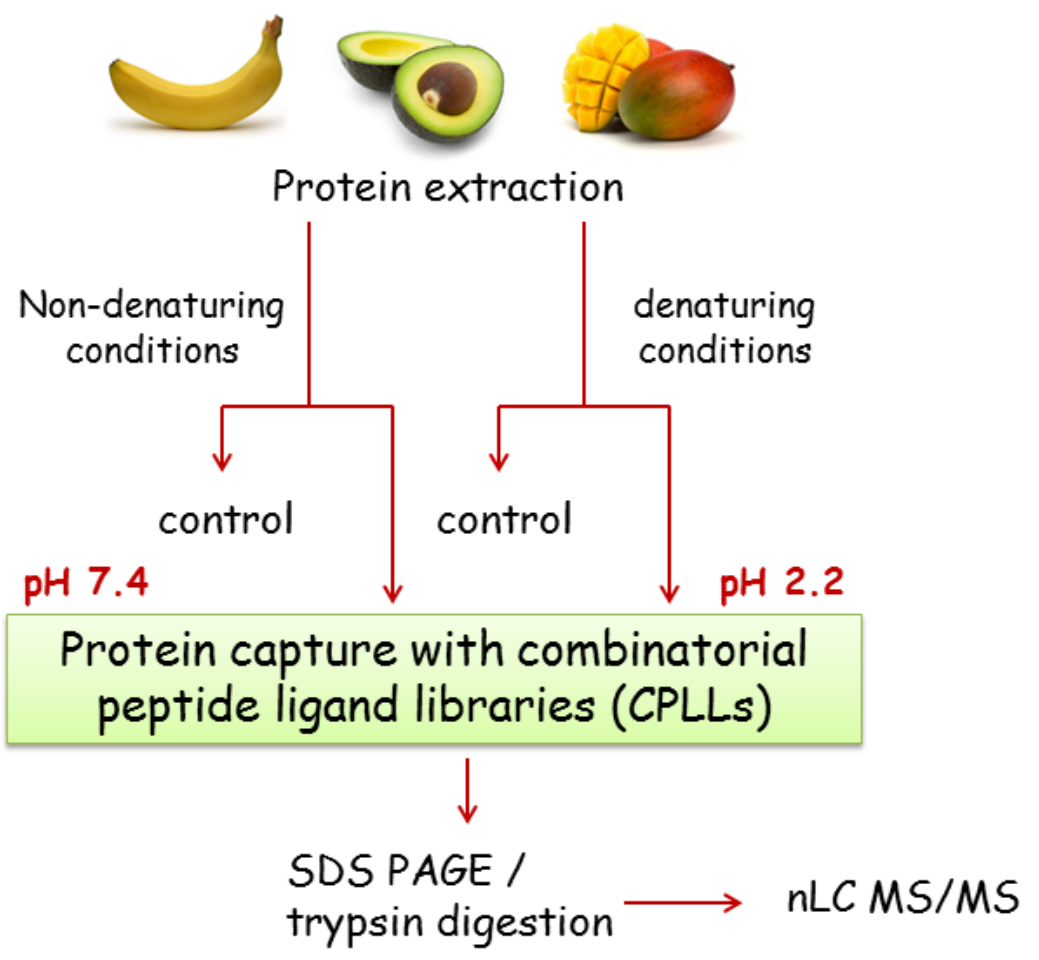


Figure 2 Banana proteomics. Upper left gel strips: SDS-PAGE of untreated vs CPLLtreated samples. Circles: Venn diagrams of total species identified under native and denaturing conditions and in the control vs CPLL-treated sample. Extreme right: plot of the functional categories against the log of number of counts of each GO term found in the filtered database.

Figure 2-Righetti et al.

\section{Banana (Musa spp.)}

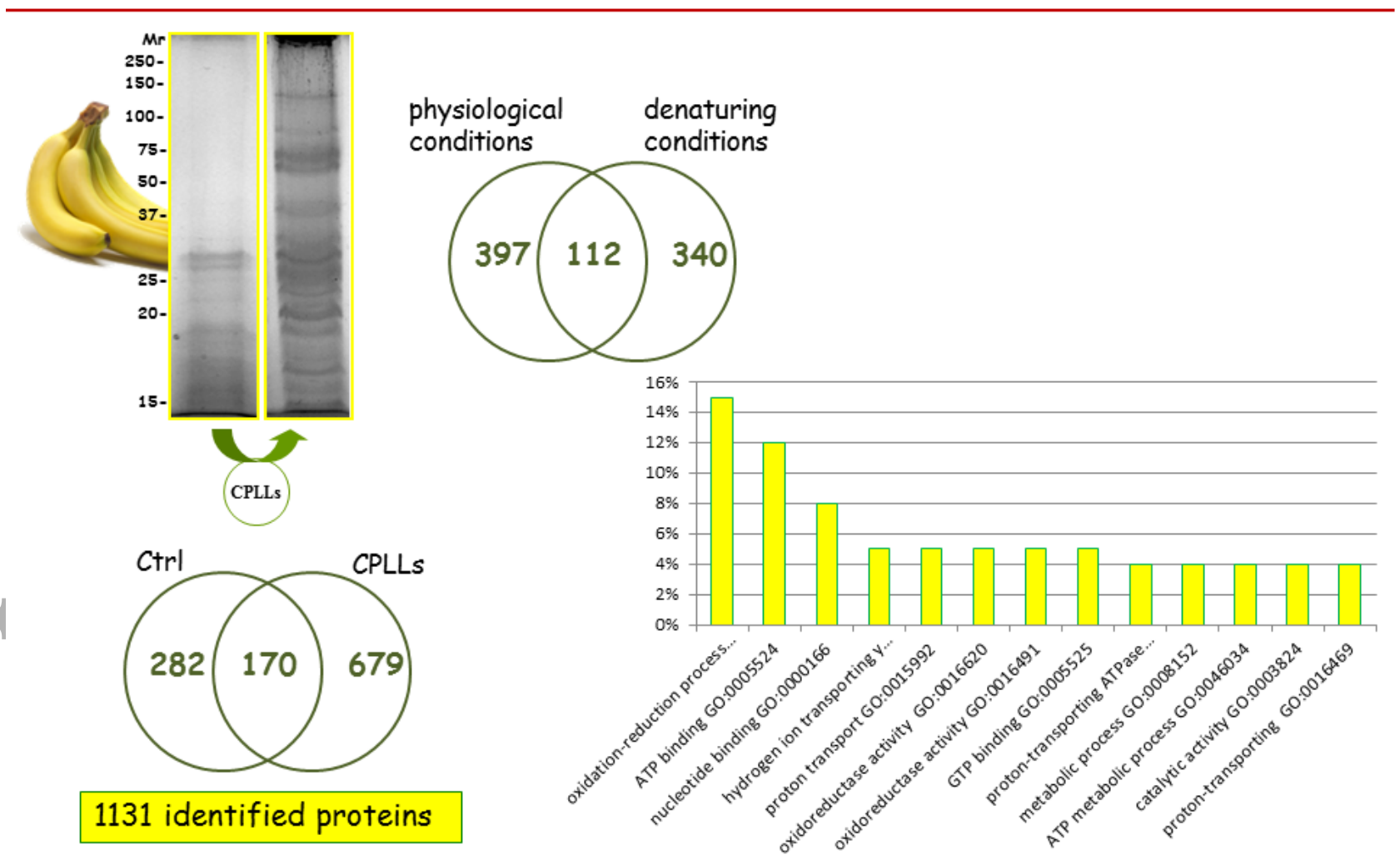


Figure 3 Avocado proteomics. Upper left gel strips: SDS-PAGE of untreated vs CPLL-treated samples. Circles: Venn diagrams of total species identified under native and denaturing conditions and in the control vs CPLL-treated sample. Lower right: plot of the functional categories against the log of number of counts of each GO terms found in the filtered database.

Figure 3-Righetti et al.

\section{Avocado (Persea Americana)}

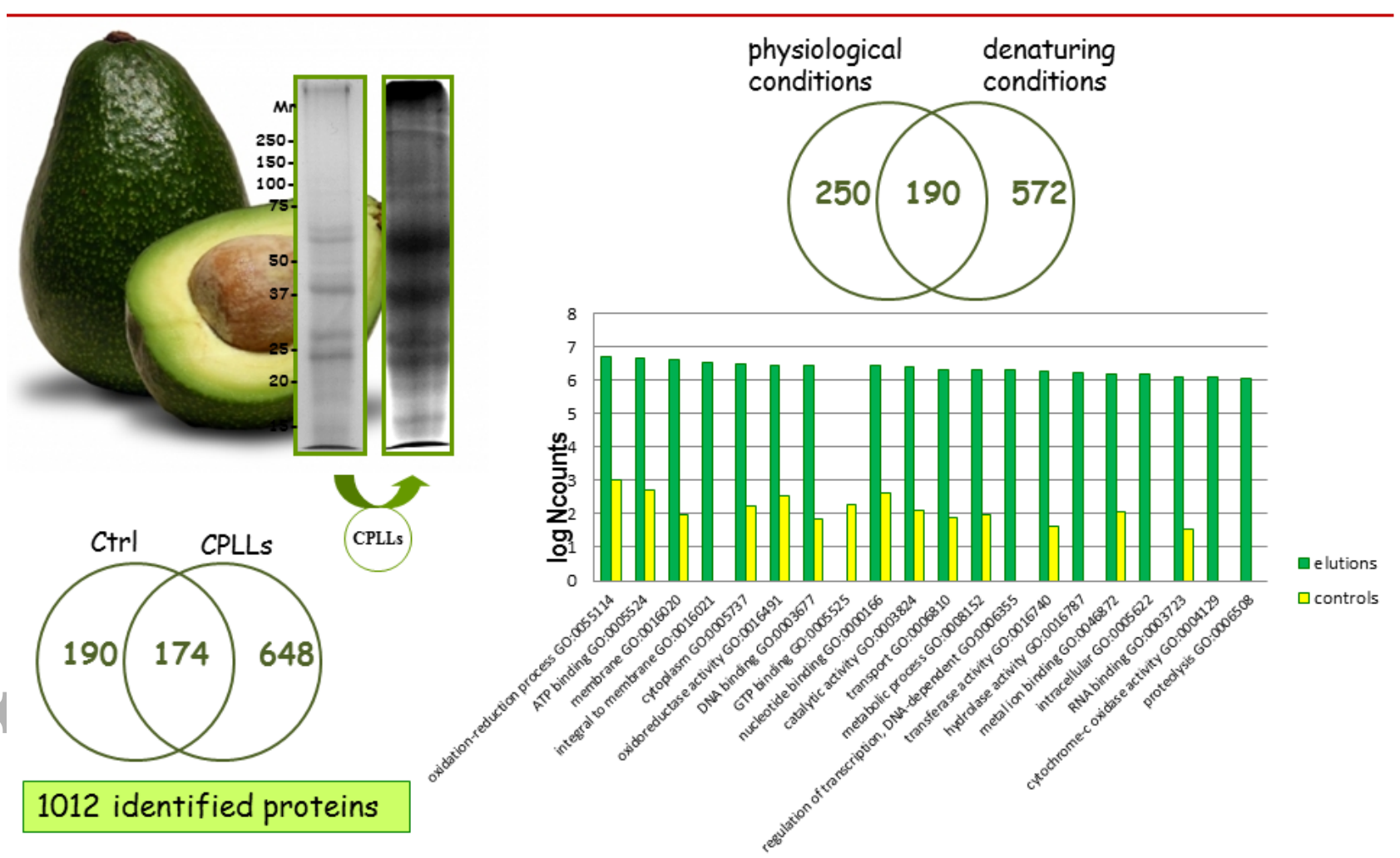


Figure 4 Mango proteomics. Extreme right gel strips: SDS-PAGE of untreated vs CPLL-treated samples of both peel and pulp. Circles: Venn diagrams of total species identified in control vs CPLL-treated samples in both peel (upper) and pulp (lower circles).

Figure 4-Righetti et al.

\section{Mango (Mangifera indica)}

Protein identification by LTQ-XL ion trap
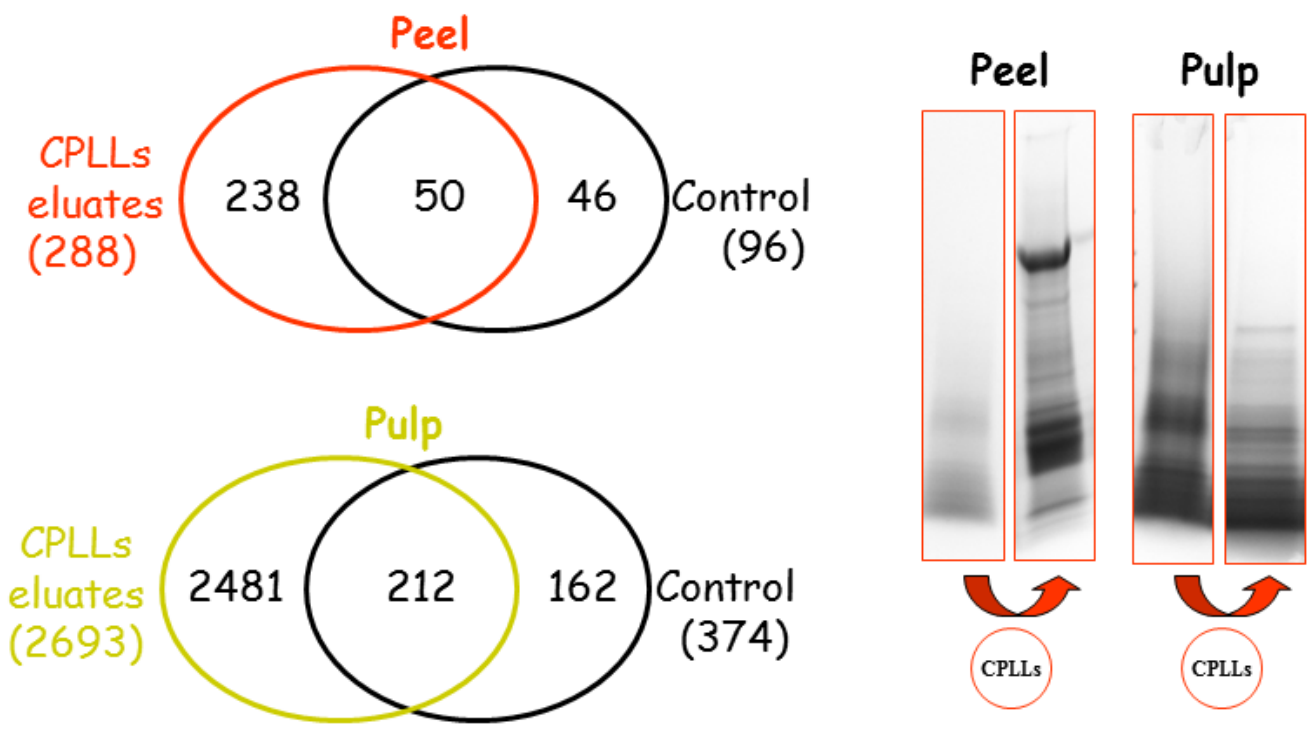
Figure 5 Plotting of the functional categories against the log of number of counts of each GO terms found in the filtered database for both peel and mango pulp. It should be noted that, in both cases, 8 categories are unique either to the peel or to the pulp, suggesting specific biological functions of these two quite different compartments.

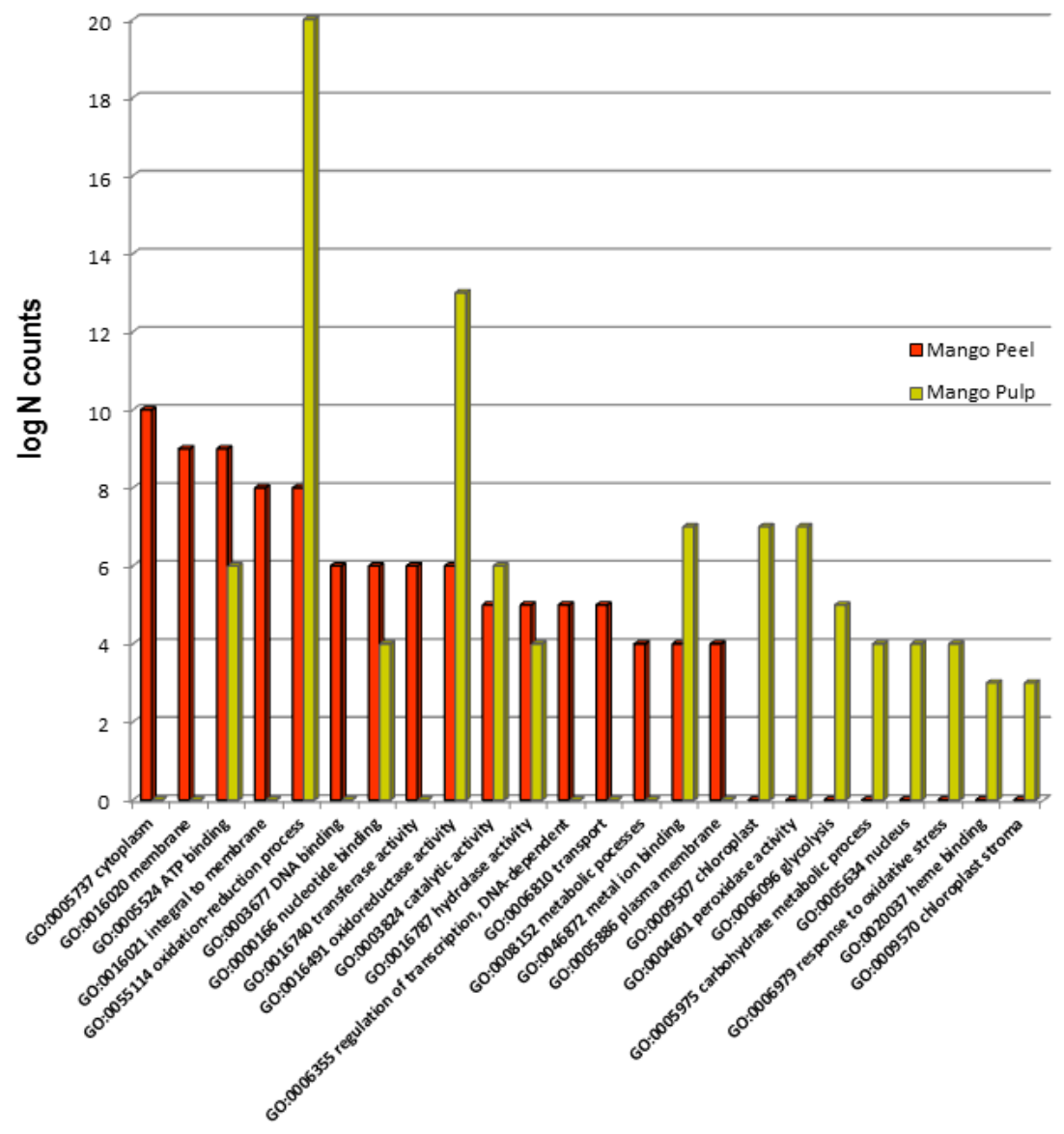

Figure 5 Righetti et al. 